\title{
Spectral asymptotics of periodic elliptic operators
}

\author{
Ola Bratteli ${ }^{1}$, \\ Palle E. T. Jørgensen ${ }^{2}$ \\ and \\ Derek W. Robinson ${ }^{3}$
}

AMS Subject Classification: 43A65, 22E45, 35H05, 22E25, 35B45, 42C05.

\begin{abstract}
We demonstrate that the structure of complex second-order strongly elliptic operators $H$ on $\mathbf{R}^{d}$ with coefficients invariant under translation by $\mathbf{Z}^{d}$ can be analyzed through decomposition in terms of versions $H_{z}$, $z \in \mathbf{T}^{d}$, of $H$ with $z$-periodic boundary conditions acting on $L_{2}\left(\mathbf{I}^{d}\right)$ where $\mathbf{I}=[0,1\rangle$. If the semigroup $S$ generated by $H$ has a Hölder continuous integral kernel satisfying Gaussian bounds then the semigroups $S^{z}$ generated by the $H_{z}$ have kernels with similar properties and $z \mapsto S^{z}$ extends to a function on $\mathbf{C}^{d} \backslash\{0\}$ which is analytic with respect to the trace norm. The sequence of semigroups $S^{(m), z}$ obtained by rescaling the coefficients of $H_{z}$ by $c(x) \rightarrow c(m x)$ converges in trace norm to the semigroup $\widehat{S}^{z}$ generated by the homogenization $\widehat{H}_{z}$ of $H_{z}$. These convergence properties allow asymptotic analysis of the spectrum of $H$.
\end{abstract}

1. University of Oslo, P. B. 1053 Blindern, N-0316 Oslo 3, Norway

2. University of Iowa, Iowa City, IA-52242-1466 USA

3. Australian National University, Canberra, ACT 0200, Australia 


\section{Introduction}

We analyze complex, strongly elliptic, periodic operators $H$ on $L_{2}\left(\mathbf{R}^{d}\right)$ in the high frequency limit. We assume the coefficients of $H$ are invariant under translation by the group $\mathbf{Z}^{d}$ and demonstrate that the semigroup $S$ generated by $H$ decomposes as a direct integral of semigroups $S^{z}, z \in \mathbf{T}^{d}$, generated by versions $H_{z}$ of $H$ acting on $L_{2}\left(\mathbf{I}^{d}\right)$, where $\mathbf{I}^{d}=\mathbf{R}^{d} / \mathbf{Z}^{d}$, with $z$-periodic boundary conditions. The decomposition corresponds to a partial Fourier decomposition of $L_{2}\left(\mathbf{R}^{d}\right)$ of the type originally occurring in Bloch wave theory [Blo28] which has recently been used in wavelet theory (see, for example, [Dau92], pages 109112). It has the advantage that if $S$ has an integral kernel with the usual continuity and boundedness properties then the $S^{z}$ have kernels which inherit similar properties. Since the $S^{z}$ act on $L_{2}\left(\mathbf{I}^{d}\right)$ and $\mathbf{I}^{d}$ is bounded it follows that the $S_{t}^{z}, t>0$, are trace class operators. The spectrum of $H$ can then be analyzed by combination of decomposition theory and the spectral theory of the $S^{z}$ in terms of Bloch bands.

The spectral analysis of the elliptic operator $H$ can be approached by homogenization theory (see, for example, [BLP78] or [ZKON79]). This corresponds to scaling the period to zero. In particular if $H^{(m)}$ denotes the sequence of operators obtained from $H$ by rescaling the coefficients of $H$ by the replacement $c_{i j}(x) \rightarrow c_{i j}^{(m)}(x)=c_{i j}(m x)$ etc. then the semigroups $S^{(m)}$ generated by the $H^{(m)}$ converge in norm, on each $L_{p}\left(\mathbf{R}^{d}\right)$-space, to the semigroup $\widehat{S}$ generated by the constant coefficient homogenization $\widehat{H}$ of $H$. This is proved by a variation of methods used in our earlier paper [BBJR95] with Charles Batty and the proof is based on kernel properties. It has the remarkable consequence that the sequence of semigroups $S^{(m), z}$ obtained from rescaling the $z$-periodic semigroups $S^{z}$ converges in trace norm on $L_{2}\left(\mathbf{I}^{d}\right)$ to the homogenization of $\widehat{S}^{z}$ of the $S^{z}$. Therefore the spectrum of $\widehat{S}^{z}$ provides an asymptotic approximation to that of $S^{z}$.

In the case of pure second-order operators with real coefficients, $H=-\sum_{i, j=1}^{d} \partial_{i} c_{i j} \partial_{j}$, our results are in part motivated by geometry where the $c_{i j}$ refer to the metric tensor. If a discrete abelian group $\Gamma$ acts freely on a non-compact differentiable manifold $\mathcal{M}$ with compact quotient then Atiyah [Ati76] and Donnelly [Don81] describe the fibering of elliptic operators $D$ on $\Gamma$-covariant bundles over $\mathcal{M} / \Gamma$. Trace estimates on the corresponding operators $D^{z}$ in the fibers lead to index computations in the geometric setting. Some of our results apply to this setting with only minor modification.

In Section 2 we describe the decomposition theory for periodic operators, semigroups and kernels. In Section 3 we extend our earlier results on homogenization and then in Section 4 we discuss the general notion of spectral refinement in the high frequency limit. We conclude with some remarks on Schrödinger operators.

\section{Periodic decompositions}

In this section we examine decomposition theory for second-order elliptic operators on the complex Hilbert space $L_{2}\left(\mathbf{R}^{d}\right)$ with periodic coefficients. Throughout the section we suppose that $H$ is the maximal accretive operator associated with the sectorial form (see, 
for example, [Kat84] [RS78])

$$
h(f)=\sum_{i, j=1}^{d}\left(\partial_{i} f, c_{i j} \partial_{j} f\right)+\sum_{i=1}^{d}\left(\left(\bar{c}_{i} f, \partial_{i} f\right)+\left(\partial_{i} f, c_{i}^{\prime} f\right)\right)+\left(f, c_{0} f\right)
$$

where the $\partial_{i}=\partial / \partial x_{i}$ denote the usual partial derivatives and the domain of $h$ is $D(h)=$ $L_{2 ; 1}\left(\mathbf{R}^{d}\right)=\bigcap_{i=1}^{d} D\left(\partial_{i}\right)$. The complex-valued coefficients $c_{i j}, c_{i}, c_{i}^{\prime}, c_{0} \in L_{\infty}\left(\mathbf{R}^{d}\right)$ and the matrix of principal coefficients $C=\left(c_{i j}\right)$, which is not necessarily symmetric, is assumed to satisfy the ellipticity condition

$$
\Re C=\left(C+C^{*}\right) / 2 \geq \lambda I>0,
$$

in the sense of $d \times d$-matrices over $\mathbf{C}^{d}$, uniformly over $\mathbf{R}^{d}$. It is this condition which ensures that the form $h$ is sectorial. The least upper bound $\lambda_{C}$ of the $\lambda$ satisfying the ellipticity condition is called the ellipticity constant.

The operator $H$ automatically generates a continuous holomorphic semigroup $S$ on $L_{2}\left(\mathbf{R}^{d}\right)$ but there is little one can deduce about the action of $S$ with no further reality, symmetry or smoothness assumptions on the coefficients. It does follow by a perturbation argument [AMT94] [ER97] that $S$ leaves $L_{2}\left(\mathbf{R}^{d}\right) \cap L_{p}\left(\mathbf{R}^{d}\right)$ invariant for $p$ sufficiently close to 2 and for all $p \in[1, \infty]$ if $d=1$ or $d=2$. Then $S$ extends to a continuous semigroup on the appropriate $L_{p}$-space. But there are examples [ACT96] which show that $S$ does not necessarily extend to all the $L_{p}$-spaces if $d \geq 5$. We first use periodicity of the coefficients and then kernel bounds to analyze the action of $S$.

All subsequent estimates depend on $\lambda_{C}$ and the $L_{\infty}\left(\mathbf{R}^{d}\right)$-norms of the coefficients of $H$. In order to trace the uniformity of the estimates it is convenient to introduce $\mathcal{E}_{N}$, for each $N>0$, as the set of elliptic operators $H$ of the above type with

$$
\lambda_{C}^{-1}+\sum_{i, j=1}^{d}\left\|c_{i j}\right\|_{\infty}+\sum_{i=1}^{d}\left(\left\|c_{i}\right\|_{\infty}+\left\|c_{i}^{\prime}\right\|_{\infty}\right)+\left\|c_{0}\right\|_{\infty} \leq N
$$

In addition we use $\mathcal{E}_{N}^{0}$ to denote the subset of $\mathcal{E}_{N}$ consisting of the pure second-order operators, i.e., those with $c_{i}=c_{i}^{\prime}=c_{0}=0$. In fact the magnitude of $\operatorname{Re} c_{0}$ is not important for uniformity of most of the estimates so we could use the alternative space $\mathcal{E}_{N}^{\prime}$ consisting of those $H$ for which there is a $\mu_{N} \in \mathbf{R}$ such that $H+\mu_{N} I \in \mathcal{E}_{N}$.

It follows by an elementary estimation that there is a $\mu \geq 0$ such that

$$
\operatorname{Re} h(f) \geq-\mu\|f\|_{L_{2}\left(\mathbf{R}^{d}\right)}
$$

for all $f \in D(h)$ where the value of $\mu$ depends only on the ellipticity constant and the $L_{\infty}\left(\mathbf{R}^{d}\right)$-norms of the coefficients. Therefore, for each $N>0$ there is a $\mu_{N} \geq 0$ such that $\Re H \geq-\mu_{N} I$ uniformly for all $H \in \mathcal{E}_{N}$ where $\Re H$ denotes the self adjoint operator associated with the closed quadratic form Re $h$. Then by the addition of $\mu_{N}$ to $c_{0}$ we may assume $\Re H$ is positive for all $H \in \mathcal{E}_{N}$. The convention $\Re H \geq 0$ ensures that the semigroup $S$ generated by $H$ is contractive. The angle of the holomorphy sector of $S$ can then be estimated in terms of the coefficients. In particular $S$ is holomorphic in the interior of the sector $\Delta(\theta)=\left\{\zeta:|\arg \zeta| \leq \cot ^{-1}\left(\|\Im C\|_{\infty} / \lambda_{C}\right)\right\}$ where $\|\Im C\|_{\infty}$ denotes the $L_{\infty}\left(\mathbf{R}^{d}\right)$-norm 
of the norm of the matrix $\Im C=\left(C-C^{*}\right) / 2 i$. Thus for each $N>0$ the semigroups $S$ generated by the $H \in \mathcal{E}_{N}$ have a common open sector of holomorphy.

In addition to the general elliptic structure we assume throughout that the coefficients of $H$ are periodic, i.e.,

$$
c_{i j}(x+n)=c_{i j}(x), \quad c_{i}(x+n)=c_{i}(x), \quad \text { etc. }
$$

for all $x \in \mathbf{R}^{d}$ and $n \in \mathbf{Z}^{d}$. (We have chosen the periods equal to one for simplicity.) If $U$ denotes the unitary action of $\mathbf{R}^{d}$ by left translations on $L_{2}\left(\mathbf{R}^{d}\right)$, i.e.,

$$
(U(y) f)(x)=f(x-y)
$$

for all $f \in L_{2}\left(\mathbf{R}^{d}\right)$ and $x, y \in \mathbf{R}^{d}$, then $U(x) D(h)=D(h)$ for all $x \in \mathbf{R}^{d}$ and the periodicity (2) of the coefficients gives the invariance property $h(U(n) f)=h(f)$ for all $n \in \mathbf{Z}^{d}$. Hence $U(x) D(H)=D(H)$ and $U(n) H=H U(n)$. The periodicity of the coefficients of $H$ is reflected by the commutation relations

$$
U(n) S_{\zeta}=S_{\zeta} U(n)
$$

on $L_{2}\left(\mathbf{R}^{d}\right)$ for all $n \in \mathbf{Z}^{d}$ and all $\zeta$ in the holomorphy sector of $S$. Next we examine versions of $H$ and $S$ on $L_{2}\left(\mathbf{I}^{d}\right)$ where $\mathbf{I}=[0,1\rangle$.

First, introduce the partial derivatives $\partial_{i}^{z}$ on $L_{2}\left(\mathbf{I}^{d}\right)$ as the skew-adjoint operators of differentiation corresponding to the $z$-periodic boundary conditions, $f\left(u_{1}, \ldots, 1, \ldots, u_{d}\right)=$ $z_{i} f\left(u_{1}, \ldots, 0, \ldots, u_{d}\right)$ where the 1 and 0 are in the $i$-th position and $z_{i} \in \mathbf{T}$. Secondly, define $H_{z}$ as the maximal accretive operator on $L_{2}\left(\mathbf{I}^{d}\right)$ associated with the sectorial form

$$
h_{z}(f)=\sum_{i, j=1}^{d}\left(\partial_{i}^{z} f, c_{i j} \partial_{j}^{z} f\right)+\sum_{i=1}^{d}\left(\left(\bar{c}_{i} f, \partial_{i}^{z} f\right)+\left(\partial_{i}^{z} f, c_{i}^{\prime} f\right)\right)+\left(f, c_{0} f\right)
$$

where $D\left(h_{z}\right)=\bigcap_{i=1}^{d} D\left(\partial_{i}^{z}\right)$. Repetition of the ellipticity estimates which gave $\Re H \geq-\mu_{N} I$ then gives $\Re H_{z} \geq-\mu_{N} I$ for each $z \in \mathrm{T}^{d}$. Thus the normalization $\Re H \geq 0$ ensures the $\Re H_{z}$ are also positive. The $H_{z}$ are versions of $H$ with $z$-periodic boundary conditions and as a consequence have discrete spectrum.

Lemma 2.1 The operators $H_{z}, z \in \mathbf{T}^{d}$, have compact resolvents.

Proof First, the real part of $h_{z}$ satisfies bounds

$$
\operatorname{Re} h_{z}(f) \geq \lambda \sum_{i=1}^{d}\left\|\partial_{i} f\right\|_{L_{2}\left(\mathbf{I}^{d}\right)}^{2}-\mu\|f\|_{L_{2}\left(\mathbf{I}^{d}\right)}^{2}
$$

with $\lambda>0$ for all $f \in D\left(h_{z}\right)$. Therefore

$$
(1+\mu) I+\Re H_{z} \geq I+\lambda L_{N}
$$

where $L_{N}$ is the version of the Laplacian on $L_{2}\left(\mathbf{I}^{d}\right)$ with Neumann boundary conditions. Since $L_{N}$ has compact resolvent it follows that $\Re H_{z}$ has compact resolvent. 
Secondly, since we have the normalization convention $\Re H_{z} \geq 0$ one can represent the resolvent $\left(\lambda I+H_{z}\right)^{-1}$ as

$$
\left(\lambda I+H_{z}\right)^{-1}=\left(\lambda I+\Re H_{z}\right)^{-1 / 2}\left(I+C_{\lambda}\right)\left(\lambda I+\Re H_{z}\right)^{-1 / 2}
$$

for all $\lambda>0$ where $C_{\lambda}$ is bounded (see, for example, (3.8) in [Kat61]). Since $\left(\lambda I+\Re H_{z}\right)^{-1 / 2}$ is compact it follows that $\left(\lambda I+H_{z}\right)^{-1}$ must be compact for all $\lambda>0$.

It follows from the positivity and maximal accretivity that the $H_{z}$ generate a family of strongly continuous contraction semigroups $S^{z}$ on $L_{2}\left(\mathbf{I}^{d}\right)$ and we subsequently demonstrate that the semigroup $S$ has a decomposition in terms of the $S^{z}$. We show in the next lemma that the family $z \mapsto S^{z}$ is strongly continuous. Subsequently, in Corollary 2.6, we establish under slightly stronger assumptions that $z \mapsto S^{z}$ extends to a function on $\mathbf{C}^{d} \backslash\{0\}$ which is analytic with respect to either the Hilbert-Schmidt norm or the trace norm.

Lemma 2.2 For each $z_{0} \in \mathbf{T}^{d}$ and $f \in L_{2}\left(\mathbf{I}^{d}\right)$

$$
\lim _{z \rightarrow z_{0}}\left\|S_{t}^{z} f-S_{t}^{z_{0}} f\right\|_{L_{2}\left(\mathbf{I}^{d}\right)}=0
$$

uniformly for $t$ in any finite interval of $[0, \infty)$.

Proof It is convenient for the proof to use the parametrization $z_{j}=e^{i \theta_{j}}$ with $\theta_{j} \in[-\pi, \pi]$ and to replace the $z$ indices and suffices by $\theta$.

Define the map $\varphi \in[-\pi, \pi]^{d} \mapsto V(\varphi)$ into unitaries on $L_{2}\left(\mathbf{I}^{d}\right)$ by

$$
(V(\varphi) f)(u)=e^{i u \cdot \varphi} f(u)
$$

This map is norm continuous and in particular $\|I-V(\varphi)\|_{2 \rightarrow 2} \rightarrow 0$ as $|\varphi| \rightarrow 0$. Moreover, $V(\varphi) D\left(h_{\theta}\right)=D\left(h_{\theta+\varphi}\right)$. But

$$
\partial_{j} V(\varphi)=V(\varphi)\left(\partial_{j}+i \varphi_{j}\right)
$$

Therefore

$$
h_{\theta+\varphi}(V(\varphi) f)=h_{\theta}(f)+p_{\theta, \varphi}(f)=h_{\theta, \varphi}(f)
$$

for all $f \in D\left(h_{\theta}\right)$ where $p_{\theta, \varphi}$ is a small form perturbation of $h_{\theta}$ with a relative bound which tends to zero as $|\varphi| \rightarrow 0$. This last property is an immediate consequence of (3) and (4). Thus if $S^{\theta, \varphi}$ denotes the continuous semigroup generated by the maximal accretive operator $H_{\theta, \varphi}$ associated with the form $h_{\theta, \varphi}$ one has

$$
V(\varphi)^{*} S_{t}^{\theta+\varphi} V(\varphi)=S_{t}^{\theta, \varphi}
$$

and hence $\left\|S_{t}^{\theta, \varphi}\right\|_{2 \rightarrow 2} \leq 1$ for all $\varphi$. Moreover $S_{t}^{\theta, \varphi}$ converges strongly to $S_{t}^{\theta}$ as $|\varphi| \rightarrow 0$ and the convergence is uniform for $t$ in any finite interval of $[0, \infty\rangle$. Hence

$$
\left(S_{t}^{\theta+\varphi}-S_{t}^{\theta}\right) f=V(\varphi)\left(S_{t}^{\theta, \varphi}-S_{t}^{\theta}\right) V(\varphi)^{*} f+\left(V(\varphi) S_{t}^{\theta} V(\varphi)^{*}-S_{t}^{\theta}\right) f
$$

Now the desired convergence as $|\varphi| \rightarrow 0$ follows by a simple estimate using the norm convergence of $V(\varphi) \rightarrow I$ and the strong convergence of $S_{t}^{\theta, \varphi} \rightarrow S_{t}^{\theta}$. 
Next we examine the decomposition of $S$ in terms of the $S^{z}$. by

First define the Zak transform (see [Dau92] pages 109-112) Z: $L_{2}\left(\mathbf{R}^{d}\right) \mapsto L_{2}\left(\mathbf{T}^{d} \times \mathbf{I}^{d}\right)$

$$
(Z f)(z, u)=\sum_{n \in \mathbf{Z}^{d}} z^{n} f(u-n)
$$

and $z^{n}=z_{1}^{n_{1}} z_{2}^{n_{2}} \ldots z_{d}^{n_{d}}$. If $f$ has compact support then the sum is finite and one calculates straightforwardly that

$$
\begin{aligned}
\|Z f\|_{L_{2}\left(\mathbf{T}^{d} \times \mathbf{I}^{d}\right)}^{2} & =(2 \pi)^{-d} \int_{\mathbf{T}^{d}}|d z| \int_{\mathbf{I}^{d}} d u\left|\sum_{n \in \mathbf{Z}^{d}} z^{n} f(u-n)\right|^{2} \\
& =\sum_{n \in \mathbf{Z}^{d}} \int_{\mathbf{I}^{d}} d u|f(u-n)|^{2}=\|f\|_{L_{2}\left(\mathbf{R}^{d}\right)}^{2}
\end{aligned}
$$

where $|d z|=\left|d z_{1}\right| \ldots\left|d z_{d}\right|$. Therefore $Z$ extends by continuity to an isometric map. But the inverse map is defined by

$$
\left(Z^{-1} f\right)(u-n)=(2 \pi)^{-d} \int_{\mathbf{T}^{d}}|d z| \bar{z}^{n} f(z, u)
$$

for $u \in \mathbf{R}^{d}$ and $n \in \mathbf{Z}^{d}$ and one again calculates that $Z^{-1}$ is a densely defined isometry. Hence $Z$ extends to a unitary map from $L_{2}\left(\mathbf{R}^{d}\right)$ to $L_{2}\left(\mathbf{T}^{d} \times \mathbf{I}^{d}\right)$. It is often convenient to extend the definition (5) to all $u \in \mathbf{R}^{d}$ and the resulting transformation satisfies the periodicity condition $(Z f)(z, x+n)=z^{n}(Z f)(z, x)$ for all $z \in \mathbf{T}^{d}, x \in \mathbf{R}^{d}$ and $n \in \mathbf{Z}^{d}$.

The Zak transform gives a decomposition of $L_{2}\left(\mathbf{R}^{d}\right)$,

$$
L_{2}\left(\mathbf{R}^{d}\right)=(2 \pi)^{-d} \int_{\mathbf{T}^{d}}^{\oplus}|d z| L_{2}\left(\mathbf{I}^{d}\right)_{z}=L_{2}\left(\mathbf{T}^{d}\right) \otimes L_{2}\left(\mathbf{I}^{d}\right)
$$

as a direct integral of copies of $L_{2}\left(\mathbf{I}^{d}\right)$ indexed by $z \in \mathbf{T}^{d}$ (for a description of the formalism of integral decompositions see, for example, [Dix69] or [BR87]). We refer to this as the Zak decomposition of $L_{2}\left(\mathbf{R}^{d}\right)$. In particular if $f \in L_{2}\left(\mathbf{R}^{d}\right)$ then, by Fubini's theorem, for almost all $z \in \mathbf{T}^{d}$ the function $f_{z}$ defined by

$$
f_{z}(u)=(Z f)(z, u)
$$

is in $L_{2}\left(\mathbf{I}^{d}\right)$ and (6) states that

$$
\|f\|_{L_{2}\left(\mathbf{R}^{d}\right)}^{2}=(2 \pi)^{-d} \int_{\mathbf{T}^{d}}|d z|\left\|f_{z}\right\|_{L_{2}\left(\mathbf{I}^{d}\right)}^{2}=\|Z f\|_{L_{2}\left(\mathbf{T}^{d}\right) \otimes L_{2}\left(\mathbf{I}^{d}\right)}^{2} .
$$

Note that $Z U(n) Z^{*}=M_{z^{n}}$ where $M_{z^{n}}$ is the operator of multiplication by $z^{n}$ on $L_{2}\left(\mathbf{T}^{d}\right) \otimes$ $L_{2}\left(\mathbf{I}^{d}\right)$. Thus if $A$ is any bounded operator on $L_{2}\left(\mathbf{R}^{d}\right)$ such that $A U(n)=U(n) A$ for all $n \in \mathbf{Z}^{d}$ then $M_{z^{n}} Z A Z^{*}=Z A Z^{*} M_{z^{n}}$ for all $n \in \mathbf{Z}^{d}$ and hence $Z A Z^{*}$ commutes with $L_{\infty}\left(\mathbf{T}^{d}\right) \otimes I_{L_{2}\left(\mathbf{I}^{d}\right)}$. Therefore $Z A Z^{*}$ is a decomposable operator,

$$
Z A Z^{*}=(2 \pi)^{-d} \int_{\mathbb{T}^{d}}^{\oplus}|d z| A(z)
$$


where the $A(z)$ are bounded operators on $L_{2}\left(\mathbf{I}^{d}\right)$ for $z \in \mathbf{T}^{d}$. If $A$ is an unbounded operator, or form, more care has to be taken but we will generally identify operators $A$ on $L_{2}\left(\mathbf{R}^{d}\right)$ and $Z A Z^{*}$ on $L_{2}\left(\mathbf{T}^{d}\right) \otimes L_{2}\left(\mathbf{I}^{d}\right)$. Now we argue that the operator $H$ and the semigroup $S$ decompose in this manner.

Let $f \in C_{c}^{\infty}\left(\mathbf{R}^{d}\right) \subset D(h)$ and define $f_{z}$ by (7). Since $f$ is differentiable it follows from the properties of the Zak transform that $f_{z} \in D\left(h_{z}\right)$ and $\left(\partial_{i} f\right)_{z}=\partial_{i}^{z} f_{z}$ for all $z \in \mathbf{T}^{d}$. Therefore a straightforward calculation using the periodicity of the coefficients gives

$$
h(f)=(2 \pi)^{-d} \int_{\mathbf{T}^{d}}|d z| h_{z}\left(f_{z}\right) .
$$

But if $D(h)$ is equipped with the norm $f \mapsto\left(\|f\|_{L_{2}\left(\mathbf{R}^{d}\right)}^{2}+\operatorname{Re} h(f)\right)^{1 / 2}$ and the $D\left(h_{z}\right)$ are equipped with analogous norms then (9) extends by closure to all of $D(h)$, by use of (8). Thus if $f \in D(h)$ then the family $z \in \mathbf{T}^{d} \mapsto f_{z}$ is $|d z|$-almost everywhere in $D\left(h_{z}\right)$ and

$$
H=(2 \pi)^{-d} \int_{\mathbf{T}^{d}}^{\oplus}|d z| H_{z}
$$

in the sense of direct integral decompositions of closed sectorial forms.

One has a similar decomposition of the semigroup.

Theorem 2.3 Let $S^{z}, z \in \mathrm{T}^{d}$, denote the continuous contraction semigroups generated by the periodic subelliptic operators $H_{z}$ on $L_{2}\left(\mathbf{I}^{d}\right)$ with z-periodic boundary conditions. Then the semigroup $S$ generated by $H$ on $L_{2}\left(\mathbf{R}^{d}\right)$ has the integral decomposition

$$
S_{t}=(2 \pi)^{-d} \int_{\mathbf{T}^{d}}^{\oplus}|d z| S_{t}^{z}
$$

corresponding to the Zak decomposition of $L_{2}\left(\mathbf{R}^{d}\right)$.

Proof The proof is relatively straightforward but requires several approximation techniques and some standard measure theoretic arguments. We sketch the main ideas.

First it is convenient to assume the coefficients of $H$ are $C^{\infty}$-functions. This ensures that $C_{c}^{\infty}\left(\mathbf{R}^{d}\right)$ is a core of $H$. Then this smoothness assumption is removed by a limiting argument.

Now if $f \in C_{c}^{\infty}\left(\mathbf{R}^{d}\right)$ one has $(H f)_{z}=H_{z} f_{z}$ and hence

$$
\left(\lambda I+H_{z}\right)^{-1} g_{z}=\left((\lambda I+H)^{-1} g\right)_{z}
$$

for all $\lambda>0$ and all $g$ in the dense set $D_{\lambda}=(\lambda I+H)\left(C_{c}^{\infty}\left(\mathbf{R}^{d}\right)\right)$. Therefore

$$
\left\|(\lambda I+H)^{-1} g\right\|_{L_{2}\left(\mathbf{R}^{d}\right)}^{2}=(2 \pi)^{-d} \int_{\mathbf{T}^{d}}|d z|\left\|\left(\lambda I+H_{z}\right)^{-1} g_{z}\right\|_{L_{2}\left(\mathbf{I}^{d}\right)}^{2}
$$

for all $g \in D_{\lambda} \subset C_{c}^{\infty}\left(\mathbf{R}^{d}\right)$. This relation then extends to all $g \in L_{2}\left(\mathbf{R}^{d}\right)$ and $\left(\lambda I+H_{z}\right)^{-1} g_{z} \in$ $L_{2}\left(\mathbf{I}^{d}\right)$ for all $z$ in a set $\Omega_{\lambda}$ with $|d z|$-measure equal to one. Then by iteration and a diagonalization argument

$$
\left\|\left(I+t_{i} H / n\right)^{-n} g\right\|_{L_{2}\left(\mathbf{R}^{d}\right)}^{2}=(2 \pi)^{-d} \int_{\mathbf{T}^{d}}|d z|\left\|\left(I+t_{i} H_{z} / n\right)^{-n} g_{z}\right\|_{L_{2}\left(\mathbf{I}^{d}\right)}^{2}
$$


and $\left(I+t_{i} H_{z} / n\right)^{-n} g_{z} \in L_{2}\left(\mathbf{I}^{d}\right)$ for all rational $t_{i}$, all positive integers $n$ and all $z$ in a set $\Omega$ with $|d z|$-measure equal to one. But the left hand side converges to $\left\|S_{t_{i}} g\right\|_{L_{2}\left(\mathbf{R}^{d}\right)}^{2}$ as $n \rightarrow \infty$ and in addition $\left\|\left(I+t_{i} H_{z} / n\right)^{-n} g_{z}\right\|_{L_{2}\left(\mathbf{I}^{d}\right)}^{2} \rightarrow\left\|S_{t_{i}}^{z} g_{z}\right\|_{L_{2}\left(\mathbf{I}^{d}\right)}^{2}$ for all $z \in \Omega$. Therefore, by the Lebesgue dominated convergence theorem,

$$
\left\|S_{t_{i}} g\right\|_{L_{2}\left(\mathbf{R}^{d}\right)}^{2}=(2 \pi)^{-d} \int_{\mathbf{T}^{d}}|d z|\left\|S_{t_{i}}^{z} g_{z}\right\|_{L_{2}\left(\mathbf{I}^{d}\right)}^{2}
$$

and $S_{t_{i}}^{z} g_{z} \in L_{2}\left(\mathbf{I}^{d}\right)$ for all $z \in \Omega$ and all rational $t_{i}$. Hence by continuity of $S$ and the $S^{z}$ one has

$$
\left\|S_{t} g\right\|_{L_{2}\left(\mathbf{R}^{d}\right)}^{2}=(2 \pi)^{-d} \int_{\mathbf{T}^{d}}|d z|\left\|S_{t}^{z} g_{z}\right\|_{L_{2}\left(\mathbf{I}^{d}\right)}^{2}
$$

for all $g \in L_{2}\left(\mathbf{R}^{d}\right)$ and $S_{t}^{z} g_{z} \in L_{2}\left(\mathbf{I}^{d}\right)$ for all $t \geq 0$ and all $z$ in a set $\Omega$ with $|d z|$-measure equal to one.

Finally, if the coefficients of $H$ are only measurable, one can approximate $H$ by a sequence of elliptic operators $H_{n}$ with $C^{\infty}$-coefficients obtained by regularization of the coefficients of $H$. Specifically the coefficients of $H$ are replaced by

$$
c_{i j}^{(n)}(x)=n^{d} \int_{\mathbf{R}^{d}} d y \tau(n y) c_{i j}(x-y)
$$

etc. where $\tau$ is a positive $C^{\infty}$-function with integral one. Then the ellipticity constants of the $H_{n}$ are bounded below by the ellipticity constant of $H$ and the $L_{\infty}$-norms of the regularized coefficients are bounded above by the $L_{\infty}$-norms of the unregularized coefficients. Thus if $H \in \mathcal{E}_{N}$ then $H_{n} \in \mathcal{E}_{N}$. Moreover, the sequence of semigroups $S^{(n)}$ generated by the $H_{n}$ converges strongly to $S$ on $L_{2}\left(\mathbf{R}^{d}\right)$ (see [ER96], Proposition 2.6). In particular

$$
\lim _{n \rightarrow \infty}\left\|S_{t}^{(n)} f-S_{t} f\right\|_{L_{2}\left(\mathbf{R}^{d}\right)}=0
$$

for all $f \in L_{2}\left(\mathbf{R}^{d}\right)$ uniformly for $t$ in finite intervals of $[0, \infty)$. But the same regularization procedure can be applied to the $H_{z}$ and one concludes that one also has strong convergence of the corresponding semigroups $S^{(n), z}$ to the $S^{z}$. All the estimates required in the regularization argument are independent of the particular choice of boundary condition. Therefore arguing as before one can deduce that

$$
\left\|S_{t}^{(n)} g\right\|_{L_{2}\left(\mathbf{R}^{d}\right)}^{2}=(2 \pi)^{-d} \int_{\mathbf{T}^{d}}|d z|\left\|S_{t}^{(n), z} g_{z}\right\|_{L_{2}\left(\mathbf{I}^{d}\right)}^{2}
$$

for all $g \in L_{2}\left(\mathbf{R}^{d}\right), n \in \mathbf{N}$ and $t>0$. Moreover, $S_{t}^{(n), z} g_{z} \in L_{2}\left(\mathbf{I}^{d}\right)$ for all $t \geq 0$, all $n \in \mathbf{N}$ and all $z$ in a set $\Omega$ with $|d z|$-measure equal to one. Taking the limit $n \rightarrow \infty$ then gives the required decomposition of $S$.

Next we assume that the action of the semigroup $S$ is given by a Hölder continuous integral kernel $K$ satisfying Gaussian upper bounds uniformly for $H$ in each $\mathcal{E}_{N}$. Specifically we assume that for each $N>0$ the semigroup $S$ generated by $H \in \mathcal{E}_{N}$ has a kernel $K$ such that

$$
\left(S_{\zeta} f\right)(x)=\int_{\mathbf{R}^{d}} d y K_{\zeta}(x ; y) f(y)
$$

for all $f \in L_{2}\left(\mathbf{R}^{d}\right)$ and all $\zeta$ in the sector of holomorphy of $S$ satisfying the following properties: 
1. there exist $a, b>0, \omega \geq 0$ and $\theta \in\langle 0, \pi / 2\rangle$ such that

$$
\left|K_{\zeta}(x ; y)\right| \leq a|\zeta|^{-d / 2} e^{\omega|\zeta|} e^{-b|x-y|^{2} /|\zeta|}
$$

for all $x, y \in \mathbf{R}^{d}$ and $\zeta \in \Delta(\theta)$,

2. in addition there exists $\nu \in\langle 0,1]$ such that

$$
\left|K_{\zeta}\left(x-x^{\prime} ; y-y^{\prime}\right)-K_{\zeta}(x ; y)\right| \leq a|\zeta|^{-d / 2} e^{\omega|\zeta|}\left(\frac{\left|x^{\prime}\right|+\left|y^{\prime}\right|}{|\zeta|^{1 / 2}}\right)^{\nu} e^{-b|x-y|^{2} /|\zeta|}
$$

for all $x, x^{\prime}, y, y^{\prime} \in \mathbf{R}^{d}$ and all $\zeta \in \Delta(\theta)$ with $\left|x^{\prime}\right|+\left|y^{\prime}\right| \leq|\zeta|^{1 / 2}$,

with both bounds uniform for $H \in \mathcal{E}_{N}$. The uniformity of these bounds will be often used in the sequel.

The $K_{\zeta}$ satisfy semigroup composition properties which reflect the corresponding properties of $S$. Moreover the periodicity of the coefficients is reflected in the periodicity of the kernel,

$$
K_{\zeta}(x ; y+n)=K_{\zeta}(x-n ; y)
$$

for all $x, y \in \mathbf{R}^{d}$ and $n \in \mathbf{Z}^{d}$. The adjoint semigroup $S^{*}$ has a similar kernel $K^{*}$ related to $K$ by $K_{t}^{*}(x ; y)=\overline{K_{t}(y ; x)}$.

One immediate consequence of the Gaussian bounds on the kernel is that $S$ leaves $L_{2}\left(\mathbf{R}^{d}\right) \cap L_{p}\left(\mathbf{R}^{d}\right)$ invariant for each $p \in[1, \infty]$ and hence extends to a continuous semigroup, also denoted by $S$, on each of the spaces $L_{p}\left(\mathbf{R}^{d}\right)$. A less obvious implication is the continuity estimate

$$
\left|K_{t-t^{\prime}}(x ; y)-K_{t}(x ; y)\right| \leq a^{\prime}\left|t^{\prime}\right| t^{-1-d / 2} e^{\omega t} e^{-b^{\prime}|x-y|^{2} /|t|}
$$

which holds for all $x, y \in \mathbf{R}^{d}$ and $t>0$ with $\left|t^{\prime}\right| \leq t / 2$. These bounds follow from the Duhamel formula

$$
K_{t}(x ; y)-K_{t-t^{\prime}}(x ; y)=\int_{t-t^{\prime}}^{t} d s \frac{d}{d s} K_{s}(x ; y)
$$

and the Cauchy representation

$$
K_{s}(x ; y)=(2 \pi i)^{-1} \int_{C_{r}(s)} d \zeta \frac{K_{\zeta}(x ; y)}{s-\zeta}
$$

where the integral is over a circle $C_{r}(s)$ of radius $r$ centred at $s$. The estimates are automatically uniform for $H \in \mathcal{E}_{N}$.

The existence of a Hölder continuous Gaussian kernel, in the above sense, is not an automatic consequence of our assumptions but it does follow from some weak additional hypotheses, e.g., if one of the following three conditions is satisfied,

1. if $d=1$ or 2 ,

2. if the principal coefficients $c_{i j}$ are real,

3. if $d \geq 3$ and the $c_{i j}$ are complex but uniformly continuous. 
On the other hand no such kernel exists in general if $d \geq 5$.

Results of this nature for low dimensions are well known but recent discussions of Gaussian bounds of the above type have been given in [AMT94] and [ER97]. The results for real coefficients are a variation of classic work of Nash [Nas58] and De Giorgi [Gio57]. A more recent description of the Nash approach is given in [FS86] and De Giorgi's approach is described in [Gia93]. The situation for complex, uniformly continuous, $c_{i j}$ is covered in [Aus96] and [ER96]. The pathologies of high dimensions are related to those of systems of elliptic operators. A variety of counterexamples in the latter setting is given in [Gia83]. The failure of Gaussian bounds for $d \geq 5$ is discussed in [ACT96]. Note that if one has the bounds (10) and (11) for complex coefficients and real $\zeta$ then they follow for complex $\zeta$ in a small sector by 'rotation': the replacement $t \rightarrow t e^{i \theta}$ corresponds to the replacement $c_{i j} \rightarrow c_{i j} e^{i \theta}$ etc.

The kernel properties transfer from $S$ to the $S^{z}$.

Theorem 2.4 Assume the semigroup $S$ has a Hölder continuous kernel $K$ satisfying Gaussian bounds. Then each $S^{z}$ has an integral kernel $K^{z}$ given by the Zak transform,

$$
K_{\zeta}^{z}(u ; v)=\sum_{n \in \mathbf{Z}^{d}} z^{n} K_{\zeta}(u ; v+n)=\sum_{n \in \mathbf{Z}^{d}} z^{n} K_{\zeta}(u-n ; v)
$$

of $K$.

The $K^{z}$ are jointly Hölder continuous and satisfy bounds

$$
\begin{aligned}
\left|K_{\zeta}^{z}(u ; v)\right| & \leq a(1 \wedge|\zeta|)^{-d / 2} e^{\omega|\zeta|} e^{-b|u-v|^{2} /|\zeta|} \\
\left|K_{\zeta}\left(u-u^{\prime} ; v-v^{\prime}\right)-K_{\zeta}(u ; v)\right| & \leq a(1 \wedge|\zeta|)^{-d / 2} e^{\omega|\zeta|}\left(\frac{\left|u^{\prime}\right|+\left|v^{\prime}\right|}{|\zeta|^{1 / 2}}\right)^{\nu} e^{-b|u-v|^{2} /|\zeta|}
\end{aligned}
$$

for all $u, v, u^{\prime}, v^{\prime} \in \mathbf{I}^{d}$ and $\zeta \in \Delta(\theta)$ with $\left|u^{\prime}\right|+\left|v^{\prime}\right| \leq|\zeta|^{1 / 2}$ and for all $z \in \mathbf{T}^{d}$, uniformly for $H \in \mathcal{E}_{N}$.

Proof We give the proof for $\zeta=t>0$.

First define functions $K^{z}$ by (13). Then the $K^{z}$ are jointly Hölder continuous and satisfy the Gaussian bounds on $\mathbf{I}^{d}$ as a simple consequence of the definition and the properties of $K$. Therefore one can define bounded operators $\widetilde{S}_{t}^{z}$ on $L_{2}\left(\mathbf{I}^{d}\right)$, or on $L_{p}\left(\mathbf{I}^{d}\right)$, by

$$
\left(\widetilde{S}_{t}^{z} f\right)(u)=\int_{\mathbf{I}^{d}} d v K_{t}^{z}(u ; v) f(v)
$$

But

$$
\begin{aligned}
\int_{\mathbf{I}^{d}} d v K_{s}^{z}(u ; v) K_{t}^{z}(v ; w) & =\sum_{n, m \in \mathbf{Z}^{d}} z^{n+m} \int_{\mathbf{I}^{d}} d v K_{s}(u ; v+n) K_{t}(v+n ; w+n+m) \\
& =\sum_{p \in \mathbf{Z}^{d}} z^{p} \int_{\mathbf{R}^{d}} d x K_{s}^{z}(u ; x) K_{t}(x ; w+p)=K_{s+t}^{z}(u ; w)
\end{aligned}
$$


where the last identification uses the semigroup property of the kernel $K$. Hence the $\widetilde{S}_{t}^{z}$ form a semigroup $\widetilde{S}^{z}$ with continuity properties similar to those of $S$. Thus it suffices to prove that $\widetilde{S}_{t}^{z}=S_{t}^{z}$

Let $f \in C_{c}\left(\mathbf{R}^{d}\right)$ then $f_{z} \in L_{2}\left(\mathbf{I}^{d}\right)$ and

$$
\begin{aligned}
\left(\widetilde{S}_{t}^{z} f_{z}\right)(u) & =\sum_{n \in \mathbf{Z}^{d}} z^{n} \int_{\mathbf{I}^{d}} d v K_{t}^{z}(u ; v) f(v-n) \\
& =\sum_{n, m \in \mathbf{Z}^{d}} z^{n+m} \int_{\mathbf{I}^{d}} d v K_{t}(u ; v+m) f(v-n) \\
& =\sum_{n, p \in \mathbf{Z}^{d}} z^{p} \int_{\mathbf{I}^{d}} d v K_{t}(u-p ; v-n) f(v-n)=\left(S_{t} f\right)_{z}(u)
\end{aligned}
$$

Therefore, from (8), one obtains

$$
\left\|S_{t} f\right\|_{L_{2}\left(\mathbf{R}^{d}\right)}^{2}=(2 \pi)^{-d} \int_{\mathbf{T}^{d}}|d z|\left\|\left(S_{t} f\right)_{z}\right\|_{L_{2}\left(\mathbf{I}^{d}\right)}^{2}=(2 \pi)^{-d} \int_{\mathbf{T}^{d}}|d z|\left\|\widetilde{S}_{t}^{z} f_{z}\right\|_{L_{2}\left(\mathbf{I}^{d}\right)}^{2}
$$

and this relation extends to all $f \in L_{2}\left(\mathbf{R}^{d}\right)$ by closure. But this means that $S$ has a direct integral decomposition in terms of the semigroups $\widetilde{S}^{z}$. Then by Theorem 2.3 it follows that $\widetilde{S}_{t}^{z}=S_{t}^{z}$ for $|d z|$-almost all $z$. Finally the equality of the semigroups follows from the continuity of $z \mapsto S_{t}^{z}$ given by Lemma 2.2 and the continuity of $z \mapsto \widetilde{S}_{t}^{z}$ which is an easy consequence of the definition of $\widetilde{S}^{z}$ and the bounds on the kernel $K$.

The kernel bounds imply that the operators $S_{\zeta}^{z}$, which are compact as a consequence of Lemma 2.1, are in fact Hilbert-Schmidt. Then the semigroup property implies they are trace class. Let $\|\cdot\|_{T r}$ and $\|\cdot\|_{H S}$ denote the trace norm and Hilbert-Schmidt norm respectively.

Lemma 2.5 Let $S=\left\{S_{t}\right\}_{t \geq 0}$ be a semigroup of bounded operators on a Hilbert space. Then

$$
\left\|S_{t}\right\|_{T r} \leq\left\|S_{t / 2}\right\|_{H S}^{2}
$$

for all $t>0$. Moreover if $S$ and $T$ are two such semigroups then

$$
\left\|S_{t}-T_{t}\right\|_{T r} \leq\left(\left\|S_{t / 2}\right\|_{H S}+\left\|T_{t / 2}\right\|_{H S}\right)\left\|S_{t / 2}-T_{t / 2}\right\|_{H S}
$$

for all $t>0$.

Proof The first statement of the lemma means that if $S_{t}$ is Hilbert-Schmidt for all $t>0$ then it is also trace class and the norm bounds are valid. The second statement is interpreted in a similar manner.

The bounds follow from the identities $S_{t}=S_{t / 2} S_{t / 2}$ and

$$
S_{t}-T_{t}=S_{t / 2}\left(S_{t / 2}-T_{t / 2}\right)+\left(S_{t / 2}-T_{t / 2}\right) T_{t / 2}
$$

together with the observation that

$$
\|A B\|_{T r} \leq\|A\|_{H S}\|B\|_{H S}
$$


for any pair of Hilbert-Schmidt operators.

Now one can estimate the trace norm as follows.

$$
\begin{aligned}
\left\|S_{2 \zeta}^{z}\right\|_{T r} & \leq\left\|S_{\zeta}^{z}\right\|_{H S}^{2}=\int_{\mathbf{I}^{d}} d u \int_{\mathbf{I}^{d}} d v\left|K_{\zeta}^{z}(u ; v)\right|^{2} \\
& \leq \sum_{n \in \mathbf{Z}^{d}} \int_{\mathbf{I}^{d}} d u \sup _{v \in \mathbf{I}^{d}}\left|K_{\zeta}(u-n ; v)\right| \sum_{m \in \mathbf{Z}^{d}} \int_{\mathbf{I}^{d}} d v \sup _{u \in \mathbf{I}^{d}}\left|K_{\zeta}(u ; v+m)\right| \\
& =\int_{\mathbf{R}^{d}} d x \sup _{v \in \mathbf{I}^{d}}\left|K_{\zeta}(x ; v)\right| \int_{\mathbf{R}^{d}} d y \sup _{u \in \mathbf{I}^{d}}\left|K_{\zeta}(u ; y)\right| \leq a^{\prime}(1 \wedge|\zeta|)^{-d} e^{2 \omega|\zeta|}
\end{aligned}
$$

where the second step uses the periodicity of the kernel, the third step follows by estimation with the Gaussian bounds and the singularity in $\zeta$ comes from the diagonal contributions $x=v$ and $u=y$ with $\omega$ the same parameter as occurs in (10).

This method of estimation gives the following substantial improvement of Lemma 2.2.

Corollary 2.6 The family $z \mapsto S^{z}$ extends to a function on $\mathbf{C}^{d} \backslash\{0\}$ which is analytic in the Hilbert-Schmidt norm, or the trace norm, on $L_{2}\left(\mathbf{I}^{d}\right)$.

Proof The extension of $S^{z}$ is defined by extension of the power series expansion (13) of the kernel $K^{z}$. The foregoing estimates then adapt to show that the extended $S^{z}$ are Hilbert-Schmidt operators. But then for $1 / R \leq|z|,\left|z_{0}\right| \leq R$ one has

$$
\begin{aligned}
\left\|S_{\zeta}^{z}-S_{\zeta}^{z_{0}}\right\|_{H S}^{2} & \leq \int_{\mathbf{I}^{d}} d u \int_{\mathbf{I}^{d}} d v\left(\sum_{n \in \mathbf{Z}^{d}}\left|z^{n}-z_{0}^{n}\right|\left|K_{\zeta}(u-n ; v)\right|\right)^{2} \\
& \leq\left|z-z_{0}\right|^{2} \sum_{n \in \mathbf{Z}^{d}} n R^{n} \int_{\mathbf{I}^{d}} d u \sup _{v \in \mathbf{I}^{d}}\left|K_{\zeta}(u-n ; v)\right| \\
& \leq a^{\prime}\left|z-z_{0}\right|^{2}(1 \wedge|\zeta|)^{-d} e^{\omega^{\prime}|\zeta|} m R^{m} \int_{\mathbf{I}^{d}} d v \sup _{u \in \mathbf{I}^{d}}\left|K_{\zeta}(u ; v+m)\right|
\end{aligned}
$$

where the last estimate relies on the Gaussian bounds. This establishes the analyticity with respect to the Hilbert-Schmidt norm. The analyticity with respect to the trace norm $\|\cdot\|_{T r}$ is a consequence of the preceding lemma. The proof of the corollary is completed by setting $S=S^{z}$ and $T=S^{z_{0}}$ in the lemma and using the Hilbert-Schmidt estimates.

Finally note the kernel $K$ is pointwise positive if and only if the coefficients of $H$ are real. But even in this situation the $K^{z}$ are not positive except in the purely periodic case $z=1$. In fact the kernels are complex for $z \neq \pm 1$.

\section{$3 \quad$ Asymptotic properties}

In this section we examine asymptotic properties of the periodic second-order operators $H$ considered in Section 2. First we reformulate some of the results obtained in [BBJR95] for 
periodic operators on $\mathbf{R}^{d}$ and then we use the decomposition theory to deduce asymptotic properties of the operators on $\mathbf{I}^{d}$. Since we did not consider complex operators nor operators with lower order terms the proofs of [BBJR95] require some adaptation. Throughout this section we assume the semigroups $S$ generated by the elliptic operators $H$ have Hölder continuous kernels satisfying the Gaussian bounds (10) and (11) uniformly for $H \in \mathcal{E}_{N}$ and for each $N>0$.

If $m$ is a positive integer we define the rescaling $H^{(m)}$ of $H$ by the replacement $c_{i j}(x) \rightarrow$ $c_{i j}^{(m)}(x)=c_{i j}(m x)$ and $c_{i}(x) \rightarrow c_{i}^{(m)}(x)=c_{i}(m x)$ etc. Then each $H^{(m)}$ is still periodic and in fact has periods $1 / m$. Next define the homogenization $\widehat{H}$ of $H$ as the elliptic operator with constant coefficients $\widehat{c}_{i j}, \widehat{c}_{i}$, etc. defined as follows. First

$$
\widehat{c}_{i j}=\int_{\mathbf{I}^{d}} d u c_{i j}(u)-\sum_{k, l=1}^{d}\left(c_{i k}, X_{k l} c_{l j}\right)
$$

where $X_{k l}$ are the bounded operators associated with the forms

$$
x_{k l}(f)=\left(\partial_{k} f, H_{1}^{-1} \partial_{l} f\right)
$$

on $L_{2}\left(\mathbf{I}^{d}\right)$ with $\partial_{k}=\left.\partial_{k}^{z}\right|_{z=1}$ and $H_{1}=\left.H_{z}\right|_{z=1}$, i.e., with $z=(1, \ldots, 1)$. (The operators $X_{k l}$ are bounded because, by spectral theory, $H_{1}^{-1}$ is bounded on the orthogonal complement of the constant functions in $L_{2}\left(\mathbf{I}^{d}\right)$.) Secondly,

$$
\widehat{c}_{i}=\int_{\mathbf{I}^{d}} d u\left(c_{i}^{\prime}(u)+c_{i}(u)\right)-\sum_{k, l=1}^{d}\left(c_{k}, X_{k l} c_{l i}\right) .
$$

Thirdly,

$$
\widehat{c}_{0}=\int_{\mathbf{I}^{d}} d x c_{0}(x)
$$

This definition of $\widehat{H}$ coincides with that of [BLP78], pages 16 and 184, and in the case of pure second-order operators with that of [BBJR95]. Note that if $C=\left(c_{i j}\right)$ is hermitian then $\widehat{C}=\left(\widehat{c}_{i j}\right)$ is also hermitian but symmetry of $C$ does not necessarily imply symmetry of $\widehat{C}$. Nevertheless the homogenized principal coefficients may be symmetrized because the anti-symmetric part gives no contribution to $\widehat{H}$.

The $\widehat{H}$ occur as limits of the rescaled versions $H^{(m)}$ of $H$. The basic argument of homogenization theory [BLP78] establishes local weak convergence of the weak solutions $u^{(m)}$ of $\left(\lambda I+H^{(m)}\right) u^{(m)}=f$ to the solution $\hat{u}$ of $(\lambda I+\widehat{H}) \hat{u}=f$. This argument is based on the following proposition.

Proposition 3.1 There is a $\lambda_{0}>0$ such that for each $f \in L_{2}\left(\mathbf{R}^{d}\right)$ and $\lambda \geq \lambda_{0}$ the equation

$$
\left(\lambda I+H^{(m)}\right) u^{(m)}=f
$$

has a unique solution $u^{(m)} \in L_{2}\left(\mathbf{R}^{d}\right)$ satisfying bounds

$$
\left\|u^{(m)}\right\|_{L_{2}\left(\mathbf{R}^{d}\right)}+\sum_{i=1}^{d}\left\|\partial_{i} u^{(m)}\right\|_{L_{2}\left(\mathbf{R}^{d}\right)} \leq c
$$

uniformly in $m$. 
Proof The proof of the proposition in the special case $c_{i}^{\prime}=c_{i}=0$ is given in [BLP78] but the general case then follows by the same arguments once one has the a priori estimate

$$
\left\|\left(\lambda I+H^{(m)}\right) f\right\|_{L_{2}\left(\mathbf{R}^{d}\right)}^{2} \geq c\left(\|f\|_{L_{2}\left(\mathbf{R}^{d}\right)}^{2}+\sum_{i=1}^{d}\left\|\partial_{i} f\right\|_{L_{2}\left(\mathbf{R}^{d}\right)}^{2}\right)
$$

valid for all sufficiently large $\lambda$ with $c>0$ independent of $m$. But this is the $L_{2}$-Gårding inequality which follows from the observation that

$$
\left\|\left(\lambda I+H^{(m)}\right) f\right\|_{L_{2}\left(\mathbf{R}^{d}\right)}^{2} \geq \lambda^{2}\|f\|_{L_{2}\left(\mathbf{R}^{d}\right)}^{2}+2 \lambda \operatorname{Re}\left(f, H^{(m)} f\right)
$$

by the usual ellipticity estimates. Since these estimates depend only on the ellipticity constant and the $L_{\infty}$-norms of the coefficients they are uniform in $m$.

Using the proposition and the arguments of [BLP78], Sections I.2 and I.13, one then proves that the $u^{(m)}$ of the proposition converge locally to the solution $\hat{u}$ of $(\lambda I+\widehat{H}) \hat{u}=f$. Then one can use general arguments of functional analysis to convert this into strong resolvent convergence on $L_{2}\left(\mathbf{R}^{d}\right)$ ([BBJR95], page 137). This in turn implies that the semigroups $S^{(m)}$ generated by the $H^{(m)}$ are strongly convergent on $L_{2}\left(\mathbf{R}^{d}\right)$ to the semigroup $\widehat{S}$ generated by $\widehat{H}$. In fact we demonstrate below that the $H^{(m)}$ are norm resolvent convergent to $\widehat{H}$ and hence the $S^{(m)}$ are norm convergent to $\widehat{S}$ on each of the $L_{p}\left(\mathbf{R}^{d}\right)$-spaces (see Corollary 3.4).

The basic convergence property of the kernels of the rescaled operators is a version of Theorem III.4 of [ZKON79].

Lemma 3.2 If $K^{(m)}$ is the kernel corresponding to $H^{(m)}$ and $\widehat{K}$ the kernel corresponding to $\widehat{H}$ then

$$
\lim _{m \rightarrow \infty} \sup _{|x|^{2}+|y|^{2} \leq v t}\left|K_{t}^{(m)}(x ; y)-\widehat{K}_{t}(x ; y)\right|=0
$$

for all $v>0$ and $t>0$.

The lemma is established in the course of the proof of Theorem III.4 of [ZKON79]. The latter proof has to be modified to cover the current context of complex coefficients but there is only one small change needed. The first part of the proof uses the semigroup convergence discussed prior to the lemma. The second part of the proof, page 137, invokes the result of Nash which gives equicontinuity of the kernel, which is then used in combination with the Arzela-Ascoli theorem to deduce a compactness property. But the required equicontinuity now follows from the bounds (11) together with the bounds (12).

Lemma 3.2 together with the periodicity and the Gaussian bounds now give a semigroup convergence result which was contained in [BBJR95] for the case of real symmetric coefficients although it was not explicitly stated.

Proposition 3.3 Let $S^{(m)}$ and $\widehat{S}$ denote the semigroups generated by the rescaled versions $H^{(m)}$ of $H$ and the homogenized operator $\widehat{H}$. Then

$$
\lim _{m \rightarrow \infty}\left\|S_{t}^{(m)}-\widehat{S}_{t}\right\|_{L_{p}\left(\mathbf{R}^{d}\right) \rightarrow L_{p}\left(\mathbf{R}^{d}\right)}=0
$$

for all $p \in[1, \infty]$, uniformly for $t$ in compact intervals of $\langle 0, \infty\rangle$. 
Proof It suffices to prove the convergence on $L_{\infty}\left(\mathbf{R}^{d}\right)$ for the semigroups and their adjoints since the general result then follows by interpolation. But as the adjoint semigroups $S^{*}$ are of the same type as $S$ it suffices to prove the $L_{\infty}\left(\mathbf{R}^{d}\right)$ convergence. This, however, is equivalent to proving that

$$
\lim _{m \rightarrow \infty} \sup _{x \in \mathbf{R}^{d}} \int_{\mathbf{R}^{d}} d y\left|K_{t}^{(m)}(x ; y)-\widehat{K}_{t}(x ; y)\right|=0
$$

with the correct uniformity in $t$. But

$$
\int_{\mathbf{R}^{d}} d y\left|K_{t}^{(m)}(x ; y)-\widehat{K}_{t}(x ; y)\right|=\int_{\mathbf{R}^{d}} d y\left|K_{t}^{(m)}(x-n ; y)-\widehat{K}_{t}(x-n ; y)\right|
$$

for each $n \in \mathbf{Z}^{d}$ by periodicity of the kernels. Therefore the supremum in (15) can be restricted to $x$ with $\left|x_{i}\right| \leq 1$. Moreover, the Gaussian bounds on $K^{(m)}$, which are uniform in $m$ by assumption, imply that

$$
\sup _{\left|x_{i}\right| \leq 1} \int_{|y|^{2} \geq v t} d y\left|K_{t}^{(m)}(x ; y)\right| \leq a e^{-b v^{2}}
$$

with similar bounds on the integral with $\widehat{K}$. Thus for $\varepsilon, t>0$ one may choose $v$ sufficiently large that

$$
\int_{\mathbf{R}^{d}} d y\left|K_{t}^{(m)}(x ; y)-\widehat{K}_{t}(x ; y)\right| \leq \varepsilon+\int_{|x|^{2}+|y|^{2} \leq v t} d y\left|K_{t}^{(m)}(x ; y)-\widehat{K}_{t}(x ; y)\right| .
$$

Then the statement of the proposition follows from Lemma 3.2.

One immediately deduces that the resolvents of the $H^{(m)}$ converge in norm.

Corollary 3.4 The sequence $H^{(m)}$ converges to $\widehat{H}$ in the norm resolvent sense on $L_{p}\left(\mathbf{R}^{d}\right)$ for each $p \in[1, \infty]$.

This follows from Proposition 3.3 by Laplace transformation.

Next we use these estimates to examine convergence of the semigroups $S^{(m), z}$ corresponding to the rescaling $H_{z}^{(m)}$ of the operator $H_{z}$ with $z$-periodic boundary conditions.

Theorem 3.5 Let $\widehat{S}^{z}$ denote the semigroup generated by the homogenized operator $\widehat{H}$ on $L_{2}\left(\mathbf{I}^{d}\right)$ with z-periodic boundary conditions. Then

$$
\lim _{m \rightarrow \infty} \sup _{z \in \mathbb{T}^{d}}\left\|S_{t}^{(m), z}-\widehat{S}_{t}^{z}\right\|_{H S}=0
$$

and

$$
\lim _{m \rightarrow \infty} \sup _{z \in \mathbf{T}^{d}}\left\|S_{t}^{(m), z}-\widehat{S}_{t}^{z}\right\|_{T r}=0
$$

uniformly for $t$ in compact intervals of $\langle 0, \infty\rangle$. 
Proof Introduce $D_{t}^{(m)}$ by

$$
D_{t}^{(m)}(x ; y)=\left|K_{t}^{(m)}(x ; y)-\widehat{K}_{t}(x ; y)\right|
$$

for $x, y \in \mathbf{R}^{d}$. Then

$$
\begin{aligned}
\left\|S_{t}^{(m), z}-\widehat{S}_{t}^{z}\right\|_{H S}^{2} & \leq \int_{\mathbf{I}^{d}} d u \int_{\mathbf{I}^{d}} d v\left|\sum_{n \in \mathbf{Z}^{d}} D_{t}^{(m)}(u-n ; v)\right|^{2} \\
& \leq \int_{\mathbf{R}^{d}} d x \sup _{v \in \mathbf{I}^{d}} D_{t}^{(m)}(x ; v) \int_{\mathbf{R}^{d}} d y \sup _{u \in \mathbf{I}^{d}} D_{t}^{(m)}(u ; y)
\end{aligned}
$$

by the estimation procedure used to deduce (14). But the $D_{t}^{(m)}$ satisfy Gaussian bounds uniformly in $m$. Hence the contribution to the integrals for $|x|^{2} \geq v t$, or $|y|^{2} \geq v t$, can be made arbitrarily small, uniformly in $m$, by choosing $v$ sufficiently large. The remaining contributions tend to zero, however, as $m \rightarrow \infty$ by Lemma 3.2. Therefore one has the Hilbert-Schmidt convergence in the uniform sense.

Finally it follows from Lemma 2.5 that

$$
\left\|S_{t}^{(m), z}-\widehat{S}_{t}^{z}\right\|_{T r} \leq\left(\left\|S_{t / 2}^{(m), z}\right\|_{H S}+\left\|\widehat{S}_{t / 2}^{z}\right\|_{H S}\right)\left\|S_{t / 2}^{(m), z}-\widehat{S}_{t / 2}^{z}\right\|_{H S}
$$

but the Hilbert-Schmidt norms of the $S_{t / 2}^{(m), z}$ and $\widehat{S}_{t / 2}^{z}$ are bounded uniformly in $m$ and $z$ by the kernel estimate that gave (14).

The trace norm convergence of the semigroups $S^{(m), z}$ to $\widehat{S}^{z}$ is equivalent to trace norm convergence of the resolvents $\left(\lambda I+H^{(m), z}\right)^{-1}$ to $\left(\lambda I+\widehat{H}^{z}\right)^{-1}$ uniformly for $\lambda$ in compact intervals of $\langle 0, \infty\rangle$. If we specialize to self-adjoint operators, i.e., if we assume $C=\left(c_{i j}\right)$ is hermitian, $c_{i}^{\prime}=-\bar{c}_{i}$ and $c_{0}$ is real, then one can immediately deduce strong statements on convergence of the spectrum of the operators. Let $\lambda_{n}(m, z)$ denote the eigenvalues of $H^{(m), z}$ in increasing order repeated according to multiplicity and $\widehat{\lambda}_{n}(z)$ the corresponding list for $\widehat{H}^{z}$ (see (36), below). The trace norm convergence implies that the corresponding finite-dimensional eigenprojections are norm convergent to the eigenprojections of the homogenized operator and the eigenvalues are pointwise convergent, i.e., $\lambda_{n}(m, z) \rightarrow \widehat{\lambda}_{n}(z)$ as $m \rightarrow \infty$. But the trace norm estimate gives a uniform bound on the eigenvalue convergence. One has

$$
\sum_{n=0}\left|e^{-t \lambda_{n}(m, z)}-e^{-t \widehat{\lambda}_{n}(z)}\right| \leq\left\|S_{t}^{(m), z}-\widehat{S}_{t}^{z}\right\|_{T r}
$$

for all $t>0$ (see [Pow67], Section 5).

If $c_{i}^{\prime}=c_{i}=c_{0}=0$ the foregoing results can be rephrased with the aid of scaling. The advantage of the pure second-order operators is that they are homogeneous of order two under dilations. Therefore by change of variable one deduces the scaling property

$$
K_{t}(x ; y)=m^{-d} K_{m^{-2} t}^{(m)}\left(m^{-1} x ; m^{-1} y\right)
$$

of the kernels associated with $H$ and the $H^{(m)}$. One immediate implication of this scaling is that (10) is valid with $\omega=0$. This follows because (10) is valid for $K^{(m)}$ uniformly in $m$ and hence

$$
\left|K_{t}(x ; y)\right| \leq a t^{-d / 2} e^{\omega m^{-2} t} e^{-b|x-y|^{2} / t}
$$


for all $m$. In the limit $m \rightarrow \infty$ one obtains the bounds with $\omega=0$. Similarly (11) is established with $\omega=0$ and as a consequence one can take $\omega=0$ in (12) and in the Gaussian estimates on $K^{z}$. Next note that since the lower order terms are zero the kernel $\widehat{K}$ obeys the scaling relation

$$
\widehat{K}_{t}(x ; y)=m^{-d} \widehat{K}_{m^{-2} t}\left(m^{-1} x ; m^{-1} y\right)
$$

Therefore the statement of the Lemma 3.2 is equivalent to

$$
\lim _{t \rightarrow \infty} \sup _{|x|^{2}+|y|^{2} \leq v t} t^{d / 2}\left|K_{t}(x ; y)-\widehat{K}_{t}(x ; y)\right|=0
$$

for all $v>0$. But this is the conclusion of Theorem III.4 of [ZKON79] (which was rephrased as Proposition 4.2 of [BBJR95] with the unfortunate omission of the factor $t^{d / 2}$ ). Finally observe that dilations are isometrically implemented on $L_{p}\left(\mathbf{R}^{d}\right)$ by the operators

$$
(V(m) f)(x)=m^{-d / p} f\left(m^{-1} x\right)
$$

for all $f \in L_{p}\left(\mathbf{R}^{d}\right)$. Then one calculates from the homogeneity of $H$ under scaling and the definition of $H^{(m)}$ that

$$
V(m)^{*}\left(S_{t}-\widehat{S}_{t}\right) V(m)=S_{m^{-2} t}^{(m)}-\widehat{S}_{m^{-2} t}
$$

Consequently

$$
\left\|S_{t}^{(m)}-\widehat{S}_{t}\right\|_{L_{p}\left(\mathbf{R}^{d}\right) \rightarrow L_{p}\left(\mathbf{R}^{d}\right)}=\left\|S_{m^{2} t}-\widehat{S}_{m^{2} t}\right\|_{L_{p}\left(\mathbf{R}^{d}\right) \rightarrow L_{p}\left(\mathbf{R}^{d}\right)}
$$

Thus the statement of Proposition 3.3 is equivalent with the following asymptotic identification.

Corollary 3.6 If $c_{i}^{\prime}=c_{i}=c_{0}=0$ then

$$
\lim _{t \rightarrow \infty}\left\|S_{t}-\widehat{S}_{t}\right\|_{L_{p}\left(\mathbf{R}^{d}\right) \rightarrow L_{p}\left(\mathbf{R}^{d}\right)}=0
$$

for all $p \in[1, \infty]$.

This was the principal conclusion of [BBJR95]. It is equivalent to the convergence properties

$$
\begin{aligned}
& \lim _{t \rightarrow \infty} \sup _{x \in \mathbf{R}^{d}} \int_{\mathbf{R}^{d}} d y\left|K_{t}(x ; y)-\widehat{K}_{t}(x ; y)\right|=0 \\
& \lim _{t \rightarrow \infty} \sup _{y \in \mathbf{R}^{d}} \int_{\mathbf{R}^{d}} d x\left|K_{t}(x ; y)-\widehat{K}_{t}(x ; y)\right|=0
\end{aligned}
$$

of the kernel. 


\section{Spectral refinement}

The homogenization process described in Section 2 shows that the rescaled versions $H^{(m), z}$ of the $z$-periodic operators converge in a very strong sense. Hence one has good estimates on their spectral properties especially if the operators are self-adjoint. The basis of the discussion was the observation that the operators are all periodic and the scaling decreased the period. But this means that the decomposition theory of Section 2 has further refinements and the limit of the spectrum is related to this refinement. In this section we analyze the details of the refinement process in a slightly more general context.

We now consider self-adjoint operators, i.e., we assume $C=\left(c_{i j}\right)$ is hermitian, $c_{i}^{\prime}=-\bar{c}_{i}$ and $c_{0}$ is real. This ensures that $H$ is self-adjoint on $L_{2}\left(\mathbf{R}^{d}\right)$ and the operators $H_{z}$ with $z$-periodic boundary conditions are self-adjoint on $L_{2}\left(\mathbf{I}^{d}\right)$. We further assume that $H$ has a Hölder continuous Gaussian kernel so all the preceding results apply. Let $H^{M}$ and $H_{z}^{M}$ be the operators obtained from $H$ and $H_{z}$ by the replacements

$$
c_{i j}(x) \rightarrow c_{i j}^{M}(x)=c_{i j}(M x)
$$

and $c_{i}(x) \rightarrow c_{i}^{M}(x)=c_{i}(M x)$ etc. where $M$ is a $d \times d$-matrix with integer coefficients and $N=|\operatorname{det} M|>1$. Subsequently, in the case that $M$ is a multiple of the identity, we consider sequences of operators obtained by iterating this map.

The new coefficients still satisfy the periodicity condition $c_{i j}^{M}(x+n)=c_{i j}^{M}(x)$ for all $n \in \mathbf{Z}^{d}$ but they also satisfy the further periodicity property

$$
c_{i j}^{M}\left(x+M^{-1} n\right)=c_{i j}^{M}(x)
$$

for $M^{-1} n \in M^{-1}\left(\mathbf{Z}^{d}\right)$ and this leads to an immediate refinement of the Zak decomposition. Define a generalized Zak transform $Z_{M}: L_{2}\left(\mathbf{R}^{d}\right) \mapsto L_{2}\left(\mathbf{T}^{d} \times M^{-1}\left(\mathbf{I}^{d}\right)\right)$ by

$$
\left(Z_{M} f\right)(z, u)=\sum_{n \in \mathbf{Z}^{d}} z^{n} f\left(u-M^{-1} n\right)
$$

One calculates as before that $Z_{M}$ is a unitary operator when $M^{-1}\left(\mathbf{I}^{d}\right)$ is equipped with Lebesgue measure inherited from $\mathbf{R}^{d}$. One may again extend the definition of $Z_{M} f$ from $u \in M^{-1}\left(\mathbf{I}^{d}\right)$ to general $u \in \mathbf{R}^{d}$ by periodicity and one then has the identity

$$
\left(Z_{M} f\right)\left(z, x+M^{-1} n\right)=z^{n}\left(Z_{M} f\right)(z, x)
$$

for all $z \in \mathbf{T}^{d}, x \in \mathbf{R}^{d}$ and $n \in \mathbf{Z}^{d}$. This leads, as before, to a decomposition

$$
L_{2}\left(\mathbf{R}^{d}\right)=(2 \pi)^{-d} \int_{\mathbb{T}^{d}}^{\oplus}|d z| \mathcal{H}_{M}(z)
$$

where $\mathcal{H}_{M}(z) \simeq L_{2}\left(M^{-1}\left(\mathbf{I}^{d}\right)\right)$.

If $A$ is an operator on $L_{2}\left(\mathbf{R}^{d}\right)$ which commutes with the action of $M^{-1} \mathbf{Z}^{d}$ then $A$ or, more precisely, $Z_{M} A Z_{M}^{*}$ has a decomposition

$$
A=(2 \pi)^{-d} \int_{\mathbb{T}^{d}}^{\oplus}|d z| A_{M, z} .
$$


$\mathbf{Z}^{d} \subseteq M^{-1} \mathbf{Z}^{d}$ the operator $A$ also commutes with the action of $\mathbf{Z}^{d}$ and has the original Zak decomposition

$$
A=(2 \pi)^{-d} \int_{\mathbf{T}^{d}}^{\oplus}|d z| A_{z}
$$

In particular if $A=H^{M}$ then $H^{M}$ has both decompositions into $H_{M, z}^{M}$ and into $H_{z}^{M}$. We now study the connection between the two decompositions and we first demonstrate that the $Z_{M}$-decomposition is a refinement of the $Z$-decomposition, i.e., each of the Hilbert spaces $\mathcal{H}(z)$ which occurs in the $Z$-decomposition of $L_{2}\left(\mathbf{R}^{d}\right)$ decomposes into a direct sum of $N$ of the Hilbert spaces $\mathcal{H}_{M}(z)$ in such a way that the corresponding components of $H^{M}$, and the semigroup $S^{M}$ generated by $H^{M}$, decompose accordingly. Adopting the notation

$$
z^{M}=\left(e^{i \theta_{1}}, \ldots, e^{i \theta_{d}}\right)^{M}=\left(e^{i \sum_{j=1}^{d} M_{j 1} \theta_{j}}, \ldots, e^{i \sum_{j=1}^{d} M_{j d} \theta_{j}}\right)
$$

this decomposition can be described as follows:

Proposition 4.1 Each Hilbert space $\mathcal{H}(z)\left(\simeq L_{2}\left(\mathbf{I}^{d}\right)\right)$ in the Zak decomposition of $L_{2}\left(\mathbf{R}^{d}\right)$ has the decomposition

$$
\mathcal{H}(z)=\bigoplus_{\substack{w \in \mathbf{T}^{d} \\ w^{M}=z}} \mathcal{H}_{M}(w)
$$

More specifically if $F=Z f$ and $F_{M}=Z_{M} f$ with $f \in L_{2}\left(\mathbf{R}^{d}\right)$ then

$$
F(z, x)=N^{-1} \sum_{\substack{w \in \mathbf{T}^{d} \\ w^{M}=z}} F_{M}(w, x)
$$

for all $z \in \mathbf{T}^{d}$ and $x \in \mathbf{R}^{d}$.

Conversely, $\mathcal{H}_{M}(z)$ is embedded into $\mathcal{H}\left(z^{M}\right)$ by

$$
F_{M}(z, x)=\sum_{p \in \mathbf{Z}^{d} / M \mathbf{Z}^{d}} z^{-p} F\left(z^{M}, x+M^{-1} p\right)
$$

for all $z \in \mathbf{T}^{d}$ and $x \in \mathbf{R}^{d}$.

Remark 4.2 Formula (25) states that if $u \in \mathbf{I}^{d}$ and $i(u)$ is the unique element of $\mathbf{Z}^{d}$ such that $u-M^{-1}(i(u)) \in M^{-1}\left(\mathbf{I}^{d}\right)$ then

$$
F(z, u)=N^{-1} \sum_{\substack{w \in \mathbf{T}^{d} \\ w=z \\ w^{M}=z}} w^{i(u)} F_{M}(w, u-i(u))
$$

which implies (24).

Remark 4.3 If $w^{M}=z$ the projection $P$ from $\mathcal{H}(z)$ onto the subspace $\mathcal{H}_{M}(w)$ may be written explicitly as

$$
(P F)(u)=N^{-1} \sum_{p \in \mathbf{Z}^{d} / M \mathbf{Z}^{d}} w^{-p} F\left(z, u+M^{-1} p\right) .
$$

(See Remark 4.5 below.) 
Proof of Proposition 4.1 By definition

$$
\begin{aligned}
F(z, u)=\left(Z Z_{M}^{-1} F_{M}\right)(z, u) & =\sum_{n \in \mathbf{Z}^{d}} z^{n}\left(Z_{M}^{-1} F_{M}\right)(u-n) \\
& =\sum_{n \in \mathbf{Z}^{d}} z^{n}(2 \pi)^{-d} \int_{\mathbf{T}^{d}}|d w| F_{M}(w, u-n) \\
& =\sum_{n \in \mathbf{Z}^{d}} z^{n}(2 \pi)^{-d} \int_{\mathbf{T}^{d}}|d w| w^{-M n} F_{M}(w, u) .
\end{aligned}
$$

Next define the mean $R$ by

$$
\left(R F_{M}\right)(w, u)=N^{-1} \sum_{\substack{v \in \mathbf{T}^{d} \\ v^{M}=w}} F_{M}(v, u)
$$

then $R$ is the adjoint of the operator $f(z) \mapsto f\left(z^{M}\right)$ and hence

$$
F(z, u)=\sum_{n \in \mathbf{Z}^{d}} z^{n}(2 \pi)^{-d} \int_{\mathbf{T}^{d}}|d w| w^{-n}\left(R F_{M}\right)(w, u)=\left(R F_{M}\right)(z, u)
$$

and (25) is established. The decomposition (24) follows automatically (see Remark 4.2).

The converse formula (26) is a consequence of the calculation

$$
\begin{aligned}
F_{M}(z, u) & =\left(Z_{M} Z^{-1} F\right)(z, u) \\
& =\sum_{n \in \mathbf{Z}^{d}} z^{n}\left(Z^{-1} F\right)\left(u-M^{-1} n\right) \\
& =\sum_{n \in \mathbf{Z}^{d}} z^{n}(2 \pi)^{-d} \int_{\mathbf{T}^{d}}|d \zeta| F\left(\zeta, u-M^{-1} n\right) \\
& =\sum_{k \in \mathbf{Z}^{d}} \sum_{y \in M^{-1} \mathbf{Z}^{d} / \mathbf{Z}^{d}} z^{(M k+M y)}(2 \pi)^{-d} \int_{\mathbf{T}^{d}}|d \zeta| F(\zeta, u-y-k) \\
& =\sum_{k \in \mathbf{Z}^{d}} \sum_{y \in M^{-1} \mathbf{Z}^{d} / \mathbf{Z}^{d}} z^{M k} z^{M y}(2 \pi)^{-d} \int_{\mathbf{T}^{d}}|d \zeta| \zeta^{-k} F(\zeta, u-y) \\
& =\sum_{y \in M^{-1} \mathbf{Z}^{d} / \mathbf{Z}^{d}} z^{M y} F\left(z^{M}, u-y\right) \\
& =\sum_{p \in \mathbf{Z}^{d} / M \mathbf{Z}^{d}} z^{-p} F\left(z^{M}, u+M^{-1} p\right)
\end{aligned}
$$

and this completes the proof.

Corollary 4.4 The z-component $H_{z}^{M}$ of $H^{M}$ in the Z-decomposition has the further decomposition

$$
H_{z}^{M}=\bigoplus_{\substack{w \in \mathbf{T}^{d} \\ w{ }^{M}=z}} H_{M, w}^{M}
$$


corresponding to the decomposition $(24)$ of $\mathcal{H}(z)$ so that the spectrum over the point $z$ in the $Z$-decomposition is the union of the spectra of the $H_{M, w}^{M}$ over the $w$ with $w=z^{M}$ in the $Z_{M}$-decomposition.

This follows from Proposition 4.1 and the fact that $H^{M}$ is decomposable with respect to both Zak decompositions.

Remark 4.5 The operator decomposition (33) refers to the Hilbert space decomposition (24) which in turn can be made more precise by the identification of the corresponding isometric embeddings $S_{w}: F_{M}(w, \cdot) \in \mathcal{H}_{M}(w) \mapsto N^{-1 / 2} F_{M}(w, \cdot) \in \mathcal{H}(z)$ where $z=w^{M}$. The operators $S_{w}$ are well defined since

$$
F_{M}(w, x+n)=F_{M}\left(w, x+M^{-1} M n\right)=w^{M n} F_{M}(w, x)=z^{n} F_{M}(w, x)
$$

for all $x \in \mathbf{R}^{d}$ and $n \in \mathbf{Z}^{d}$. The adjoint operator $S_{w}^{*}: \mathcal{H}(z) \mapsto \mathcal{H}_{M}(w)$ is given by

$$
\left(S_{w}^{*} F\right)(z)=N^{-1 / 2} \sum_{p \in \mathbf{Z}^{d} / M \mathbf{Z}^{d}} w^{-p} F\left(z^{M}, x+M^{-1} p\right) .
$$

It can be checked that the projections $P_{w}=S_{w} S_{w}^{*}$ on $\mathcal{H}(z)$ are mutually orthogonal and

$$
\sum_{w: w^{M}=z} P_{w}=I_{\mathcal{H}(z)}
$$

The formula for $P_{w}$ is the finite Fourier transform in (28). We may use this and (33) to identify the eigenvectors corresponding to the refined spectrum. Let $\lambda(z)$ be in the spectrum of $Z H^{M} Z^{-1}$ with eigenvector $F \in \mathcal{H}(z)$. The projections $P_{w}=S_{w} S_{w}^{*}$ commute with $Z H^{M} Z^{-1}$. The $w \in \mathbf{T}^{d}$ such that $F \in\left(Z_{M} H^{M} Z_{M}^{-1}\right)(w)$ are exactly those $w$ (generically only one) such that $S_{w}^{*} F \neq 0$. This happens if and only if the corresponding conjugacy class in $\mathbf{Z}^{d} / M \mathbf{Z}^{d}$ contributes to the Fourier series expansion of $F$.

In order to analyze the spectrum in more detail we specialize the transformation $M$. We assume $M=N I$ where $N \in\{2,3, \ldots\}$ so that $\operatorname{det} M=N^{d}$ and we use $N$ as index instead of $M$, i.e., we write $Z_{N}$ instead of $Z_{M}$ etc. This special choice of $M$ corresponds to a rescaling of the type considered in Section 3 and hence the semigroups $S^{N}$ generated by the rescaled operators $H^{N}$ converge to the semigroup $\widehat{S}$ generated by the homogenization $\widehat{H}$ of $H$. We expect that for general transformations $M$ of the foregoing type the corresponding semigroups $S^{M}$ will have good convergence properties if all the eigenvalues of $M$ have modulus strictly larger than one but it appears difficult to identify their limit. Hence we restrict attention to the special case $M=N I$ and discuss the links between the spectral refinement and the homogenization limit which we obtained in Section 3.

The special choice of $M$ now gives

$$
\left(Z_{N} f\right)(z, u)=\sum_{n \in \mathbf{Z}^{d}} z^{n} f(u-n / N)
$$

for $u \in N^{-1} \mathbf{I}^{d}$ and (27) takes the form

$$
F\left(z, u+N^{-1} i\right)=N^{-d} \sum_{\substack{w \in \mathbf{T}^{d} \\ w^{N}=z}} w^{i} F_{N}(w, u)
$$


for $u \in N^{-1} \mathbf{I}^{d}, i=\left(i_{1}, \ldots, i_{d}\right)$ with $i_{k} \in\{0,1, \ldots, N-1\}$.

Let $H_{z}^{N}$ and $H_{N, z}^{N}$ denote the components of the rescaled operator $H^{N}$ in the $Z$ and $Z_{N}$ decompositions, respectively. For all $z \in \mathbf{T}^{d}$ let $\lambda_{n}(z)$ denote the eigenvalues of $H_{z}$ on $L_{2}\left(\mathbf{I}^{d}\right)$ arranged in increasing order repeated according to multiplicity. Now consider pure second-order operators, $H=-\sum_{i, j=1}^{d} \partial_{i} c_{i j} \partial_{j}$. Then by scaling the eigenvalues of $H_{N, z}^{N}$ on $L_{2}\left(N^{-1} \mathbf{I}^{d}\right)$ are $N^{2} \lambda_{n}(z)$. As a consequence of Corollary 4.4 one draws the following conclusion about the spectrum.

Corollary 4.6 Let $H$ be a pure second-order, self-adjoint, elliptic operator and let $\lambda_{n}(z)$ denote the eigenvalues of $H_{z}$ on $L_{2}\left(\mathbf{I}^{d}\right)$. The eigenvalues, counted with multiplicity, of the rescaled operator

$$
H_{z}^{N}=-\sum_{i, j=1}^{d} \partial_{i} c_{i j}(N \cdot) \partial_{j}
$$

with $z$-periodic boundary conditions on $L_{2}\left(\mathbf{I}^{d}\right)$ form the set of $N^{2} \lambda_{n}(w)$ with $n=0,1, \ldots$ and $w \in \mathbf{T}^{d}$ with $w^{N}=z$.

If we now take the limit $N \rightarrow \infty$ the semigroups $S^{N, z}$, generated by $H_{z}^{N}$ on $L_{2}\left(\mathbf{I}^{d}\right)$, converge in the trace norm, by Theorem 3.5, uniformly for $t$ in compact intervals of $\langle 0, \infty\rangle$ to the semigroup $\widehat{S}_{z}$ generated by the homogenization $\widehat{H}_{z}$ of $H_{z}$. Therefore the eigenvalues converge pointwise to those of $\widehat{H}_{z}$ and the finite-dimensional eigenprojections converge in norm. But the spectrum of $\widehat{H}_{z}$ with $z=\left(e^{i \theta_{1}}, \ldots, e^{i \theta_{d}}\right)$ is easily computed. For $z$ fixed set $\varphi_{n}(u)=e^{i(\theta-n) \cdot u}$ for $n \in \mathbf{Z}^{d}$ then

$$
\widehat{H}_{z} \varphi_{n}=\sum_{i, j=1}^{d} \widehat{c}_{i j}\left(n_{i}-\theta_{i}\right)\left(n_{j}-\theta_{j}\right) \varphi_{n}
$$

so the eigenvalues of $\widehat{H}_{z}$, counted with multiplicity, are

$$
\left\{\langle(n-\theta), \widehat{C}(n-\theta)\rangle: n \in \mathbf{Z}^{d}\right\} .
$$

Therefore one has the following asymptotic identification of the spectrum of $H_{z}$.

Corollary 4.7 If $\lambda_{n}(z)$ denotes the eigenvalues of $H_{z}$ then

$$
\lim _{N \rightarrow \infty}\left\{N^{2} \lambda_{n}(w): w^{N}=z, n=0,1, \ldots\right\}=\left\{\langle(n-\theta), \widehat{C}(n-\theta)\rangle: n \in \mathbf{Z}^{d}\right\}
$$

where the limit is in the sense of pointwise convergence of the ordered sets.

The rate of convergence of the eigenvalues can be estimated further by the trace norm estimate (16).

\section{Concluding remarks}

Adopt the general assumptions in the beginning of Section 4 , so that $H$ is self-adjoint on $L_{2}\left(\mathbf{R}^{d}\right)$. Again let $\lambda_{n}(z), z \in \mathbf{T}^{d}$, denote the eigenvalues of $H_{z}$ on $L_{2}\left(\mathbf{I}^{d}\right)$ arranged in 
increasing order repeated according to multiplicity. It then follows from Corollary 2.6 and the minimax principle that the functions $z \mapsto \lambda_{n}(z)$ are continuous on $\mathbf{T}^{d}$ and can even be shown to be piecewise analytic in a suitable sense [Kat84], Theorem VII.7.18, [Don81], Lemma 2.1. It then follows, see for example [RS78], Theorem XIII.85, that the spectrum of $H$ on $L_{2}\left(\mathbf{R}^{d}\right)$ is the union of the closed intervals

$$
B_{n}=\left\{\lambda_{n}(z): z \in \mathbf{T}^{d}\right\} .
$$

These are called the bands of the spectrum and they may, or may not, overlap and in principle they could, or could not, contain points which are eigenvalues (corresponding to open sets in $\mathbf{T}^{d}$ where $\lambda_{n}$ is constant). Because of piecewise analyticity the spectrum of $H$, apart from the possible eigenvalues, is absolutely continuous. Corollary 4.7 shows among other things that possible gaps in the bands of $H^{N}$ disappear as $N \rightarrow \infty$ and the spectrum converges to the wellknown spectrum $[0, \infty)$ of $\widehat{H}$ with multiplicities given by Fourier analysis.

One instance where $H$ has no eigenvalues is the special case of Schrödinger operators,

$$
H=-\Delta+V
$$

with periodic potentials $V$. There has been a great deal of interest in these operators since the seminal work of Bloch [Blo28] (see, for example, [DT82] [Eas73] [RS78] [Skr85] [Sun88] [Wil78]). It is known that the spectrum of $H$ on $L_{2}\left(\mathbf{R}^{d}\right)$ is absolutely continuous (see, for example, [Tho73] [Wil78] and [RS78], Theorem XIII.100). The argument is roughly the following. If $\lambda>0$ and $t>0$ are fixed, the operators $\left(\lambda I+H_{z}\right)^{-1}$ and $S_{t}^{z}$ have analytic continuations to $0<|z|<+\infty$ by the results in Section 2 . Introducing $k \in \mathbf{C}^{d}$ by $z=\exp (i k)$, these functions are entire analytic in $k$ with values in the trace class operators, by Corollary 2.6. Now if the $\lambda_{n}$, viewed as functions of $k$, are constant in an open set then $H_{z}$ has a constant eigenvalue for $z \in \mathbf{C}^{d} \backslash\{0\}$ by [Kat84] [Don81]. But then the norms $\left\|\left(\lambda I+H_{z}\right)^{-1}\right\|_{L_{2}\left(\mathbf{I}^{d}\right)}$ and $\left\|S_{t}^{z}\right\|_{L_{2}\left(\mathbf{I}^{d}\right)}$ would have positive lower bounds uniformly in $z \in \mathbf{C}^{d} \backslash\{0\}$. If, however, $H$ is a Schrödinger operator then Thomas [Tho73] shows that $\left\|\left(\lambda I+H_{z}\right)^{-1}\right\|_{L_{2}\left(\mathbf{I}^{d}\right)} \rightarrow 0$ as $k \rightarrow \infty$ through a line in the imaginary direction. The estimates in Section 2 could possibly be elaborated to reach a similar conclusion for general self-adjoint elliptic operators $H$.

Returning to the Schrödinger operator (37), we have seen that its spectrum is the union of bands

$$
B_{n}(V)=\left\{\lambda_{n}(z): z \in \mathbf{T}^{d}\right\} .
$$

If $d=1$ the $n$-th band is located in a neighbourhood of $\pi n^{2}$ and the bands may or may not overlap depending on the nature of $V$. In general the length of the gaps between bands, if such a gap exists, tends to zero as $n \rightarrow \infty$ and if $\bar{V}=\int_{0}^{1} d x V(x)$ is the mean of $V$ then the length of each gap is dominated by $2\left(\int_{0}^{1} d x(V(x)-\bar{V})^{2}\right)^{1 / 2}$ in the region $\langle\bar{V}, \infty\rangle$. Thus there are no gaps if $V$ is constant as we can also see from the direct computation $\left\{\lambda_{n}\left(e^{i \theta}\right): n \in \mathbf{N}\right\}=\left\{(k+\theta)^{2}+V: k \in \mathbf{Z}\right\}$. Conversely it can be proved that if there are no gaps then $V$ is constant. There are examples where all gaps are open. If there is precisely one gap then $V$ is a Weierstrass elliptic function. If there are only finitely many gaps then $V$ is real analytic and if all the odd gaps are absent then $V$ has period $1 / 2$ (see [Eas73] and [RS78], Section VIII.16, for a discussion of these results). 
If $d=2$ it is established in [DT82] that there exist positive $c$ and $C$ such that

$$
\left[4 \pi n-c n^{1 / 4}, 4 \pi n+c n^{1 / 4}\right] \subseteq B_{n}(V) \subseteq\left[4 \pi n-C n^{1 / 3}, 4 \pi n+C n^{1 / 3}\right] .
$$

Hence the spectrum may have a finite number of open gaps near zero but the spectrum always contains all sufficiently large $\lambda \in \mathbf{R}$. If $d=3$ this qualitative picture remains. If one defines a multiplicity function $M$ by

$$
M(\lambda)=\#\left\{n: \lambda \in B_{n}(V)\right\}
$$

it is proved in [Skr85] that there exist positive $c$ and $C$ such that

$$
c \lambda^{1 / 2}<M(\lambda)<C \lambda^{3 / 4}
$$

for large $\lambda$ and it is conjectured that $\lambda^{3 / 4}$ may be replaced by $\lambda^{1 / 2+\varepsilon}$ in the upper estimate. In this case it can also be shown that if $\|V\|_{\infty}<D$ where $D$ is a universal constant there are no gaps in the spectrum.

If $V$ is replaced by $V_{\varepsilon}=V\left(\varepsilon^{-1} \cdot\right)$ it follows from Corollary 3.4 that $H_{\varepsilon}=-\Delta+V_{\varepsilon}$ converges to $\widehat{H}=-\Delta+\bar{V}$ in the norm resolvent sense with

$$
\bar{V}=\int_{\mathbf{I}^{d}} d x V(x)
$$

Since $\bar{V}$ is constant the spectral properties of $\widehat{H}$ are the same as those of $-\Delta$ and we may formulate a corollary similar to Corollary 4.7 although the eigenvalues of $H_{N, z}$ can no longer be computed from those of $H_{z}$ by simple scaling properties. Let us finally remark that if $H_{\varepsilon}$ is an operator of the form

$$
H_{\varepsilon}=H_{0}+V_{\varepsilon}
$$

where $H_{0}$ is any elliptic operator coming from a form of the type (1), with no periodicity required, with a kernel satisfying the bounds (10) and (11) then $H_{\varepsilon} \rightarrow \widehat{H}=H_{0}+\bar{V}$ in the norm resolvent sense. This can be established by the following direct argument.

The Duhamel expansion

$$
S_{t}^{\varepsilon}-\widehat{S}_{t}=\int_{0}^{t} d s S_{s}^{\varepsilon}\left(\bar{V}-V_{\varepsilon}\right) \widehat{S}_{t-s}
$$

for the corresponding semigroups is valid on $L_{2}\left(\mathbf{R}^{d}\right)$ and for fixed $t$ the kernel of the operator in the integrand is

$$
\begin{aligned}
K(s: x ; y) & =\int_{\mathbf{R}^{d}} d u K_{s}^{\varepsilon}(x ; u)\left(\left(\bar{V}-V\left(\varepsilon^{-1} u\right)\right) \widehat{K}_{t-s}(u ; y)\right. \\
& =\sum_{n \in \mathbf{Z}^{d}} \varepsilon^{d} \int_{n+\mathbf{I}^{d}} d u K_{s}^{\varepsilon}(x ; \varepsilon u)\left((\bar{V}-V(u)) \widehat{K}_{t-s}(\varepsilon u ; y)\right.
\end{aligned}
$$

Now we use the Hölder continuity (11) to estimate each of the terms in the sum by $O\left(\varepsilon^{\nu}\right)$ when $\varepsilon u$ is close to $x$ or $y$ and use the Gaussian bounds (10) to estimate in terms of $\|\bar{V}-V\|_{\infty}$ times a Gaussian when $\varepsilon u$ is far from $x$ and $y$. These estimates are singular in 
$s$ when it is near 0 or $t$ but this can be remedied by first estimating the Duhamel integral near these limits in terms of $\left\|V-V_{\varepsilon}\right\|_{\infty}$. These estimates lead to the conclusion that

$$
\lim _{\varepsilon \rightarrow 0} \sup _{x \in \mathbf{R}^{d}} \int_{\mathbf{R}^{d}} d y|K(s: x ; y)|=0
$$

uniformly for $s$ in compact intervals of $\langle 0, t\rangle$. One then concludes that one has norm convergence of the semigroups, $\left\|S_{t}^{\varepsilon}-\widehat{S}_{t}\right\|_{L_{p}\left(\mathbf{R}^{d}\right) \rightarrow L_{p}\left(\mathbf{R}^{d}\right)} \rightarrow 0$ as $\varepsilon \rightarrow 0$ for each $p \in[1, \infty]$, uniformly for $t$ in compact intervals of $\langle 0, \infty\rangle$.

\section{Acknowledgements}

This work was carried out whilst the first two authors were visiting the Centre for Mathematics and its Applications at the ANU with financial support of the Centre. The first author also obtained travel support from the Norwegian Research Council. All three authors are indebted to Tom ter Elst for a critical reading of the manuscript and several helpful suggestions.

\section{References}

[Ati76] Aттун, M. F., Elliptic operators, discrete groups and von Neumann algebras. Astérisque 32-33 (1976).

[Aus96] Auscher, P., Regularity theorems and heat kernel for elliptic operators. $J$. London Math. Soc. 54 (1996), 284-296.

[ACT96] Auscher, P., Coulhon, T., and Tchamitchian, P., Absence de principe du maximum pour certaines équations paraboliques complexes. Colloq. Math. 71 (1996), 87-95.

[AMT94] Auscher, P., McIntosh, A., and Tchamitchian, P., Heat kernels of second order complex elliptic operators and their applications. Research Report 94-164, Maquarie University, Sydney, Australia, 1994.

[BBJR95] Batty, C. J. K., Bratteli, O., Jørgensen, P. E. T., and Robinson, D. W., Asymptotics of periodic subelliptic operators. J. Geom. Anal. 5 (1995), 427-443.

[BLP78] Bensoussan, A., Lions, J. L., and Papanicolaou, G., Asymptotic analysis for periodic structures, vol. 5 of Studies in Mathematics and its Applications. North-Holland, Amsterdam etc., 1978.

[Blo28] BLOCH, F., Uber die quantenmechanik der elektronen in kristallgittern. Zeit. für Physik 52 (1928), 555-600.

[BR87] Bratteli, O., and Robinson, D. W., Operator algebras and quantum statistical mechanics, vol. 1. Second edition. Springer-Verlag, New York etc., 1987. 
[DT82] Dahlberg, B. E. J., and Trubowitz, E., A remark on two dimensional periodic potentials. Comm. Math. Helvetici 57 (1982), 130-134.

[Dau92] Daubechies, I., Ten lectures on wavelets. CBMS Regional Conference Series in Mathematics 61. Amer. Math. Soc., Providence, 1992.

[Dix69] Dixmier, J., les algèbres d'opérateurs dans l'espace Hilbertien. Cahiers scientifiques 25. Gauthier-Villars, Paris, 1969.

[Don81] Donnelly, H., On $L_{2}$-Betti numbers for abelian groups. Can. Math. Bull. 24 (1981), 91-95.

[Eas73] Eastham, M. S. P., The spectral theory of periodic differential equations. Scottish Academic Press, Edinburgh and London, 1973.

[ER96] Elst, A. F. M. TER, and Robinson, D. W., Second-order subelliptic operators on Lie groups I: complex uniformly continuous principal coefficients. Research Report MRR 035-96, The Australian National University, Canberra, Australia, 1996.

[ER97] - High order divergence-form elliptic operators on Lie groups. Bull. Austral. Math. Soc. 55 (1997), 335-348.

[FS86] FABES, E. B., and STRoock, D. W., A new proof of Moser's parabolic Harnack inequality using the old ideas of Nash. Arch. Rat. Mech. and Anal. 96 (1986), 327-338.

[Gia83] Giaquinta, M., Multiple integrals in the calculus of variations and nonlinear elliptic systems. Annals of Mathematics Studies 105. Princeton University Press, Princeton, 1983.

[Gia93] _ Introduction to regularity theory for nonlinear elliptic systems. Lectures in Mathematics ETH Zürich. Birkhäuser Verlag, Basel etc., 1993.

[Gio57] Giongi, E. D., Sulla differenziabilità e l'analiticità delle estremali degli integrali multipli regolari. Mem. Accad. Sci. Torino cl. Sci. Fis. Mat. Nat. 3 (1957), 25-43.

[Kat61] Kato, T., Fractional powers of dissipative operators. J. Math. Soc. Japan 13 (1961), 246-274.

[Kat84] —, Perturbation theory for linear operators. Second edition, Grundlehren der mathematischen Wissenschaften 132. Springer-Verlag, Berlin etc., 1984.

[Nas58] NASH, J., Continuity of solutions of parabolic and elliptic equations. Amer. J. Math. 80 (1958), 931-954.

[Pow67] Powers, R. T., Representations of uniformly hyperfinite algebras and their associated von Neumann rings. Ann. Math. 86 (1967), 138-171. 
[RS78] REed, M., and Simon, B., Methods of modern mathematical physics IV. Analysis of operators. Academic Press, New York etc., 1978.

[Skr85] Skriganov, M. M., The spectrum band structure of the three-dimensional Schrödinger operator with periodic potential. Invent. Math. 80 (1985), 107-121.

[Sun88] SunAdA, T., Fundamental groups and Laplacians. In Geometry and analysis on manifolds, Lecture Notes in Mathematics 1339. Springer-Verlag, Berlin etc., 1988.

[Tho73] Thomas, L. E., Time dependent approach to scattering from impurities in a crystal. Commun. Math. Phys. 33 (1973), 335-343.

[Wil78] Wilcox, C. H., Theory of Bloch waves. J. d'Analyse Math. 33 (1978), 146167.

[ZKOn79] Zhikov, V. V., Kozlov, S. M., Oleinik, O. A., and Ngoan, K. T., Averaging and $G$-convergence of differential operators. Russian Math. Surveys 34 (1979), 569-147. 